\title{
ESTOQUE E CRESCIMENTO EM VOLUME, BIOMASSA, CARBONO E DIÓXIDO DE CARBONO EM FLORESTA ESTACIONAL SEMIDECIDUAL ${ }^{1}$
}

\author{
Agostinho Lopes de Souza², Aline Boina ${ }^{3}$, Carlos Pedro Boechat Soares ${ }^{2}$, Benedito Rocha Vital², Ricardo \\ de Oliveira Gaspar ${ }^{3}$ e Jacinto Moreira de Lana ${ }^{4}$
}

\begin{abstract}
RESUMO - O objetivo deste trabalho foi estimar o estoque e o crescimento em volume (V), biomassa (B), carbono (C) e dióxido de carbono $\left(\mathrm{CO}_{2}\right)$ em Floresta Estacional Semidecidual no Vale do Rio Doce, em Minas Gerais. Foram utilizados dados de inventários do estrato arbóreo (DAP $\geq 5,0 \mathrm{~cm}$ ), cujas parcelas permanentes foram medidas em 2002 e 2007, em estágios médio (Mata 1) e avançado (Mata 2) de regeneração da vegetação secundária. Com base no inventário de 2002, foram selecionadas espécies que apresentavam maiores percentuais em volume e no mínimo cinco indivíduos para determinar as densidades básicas da madeira e da casca. A média da densidade básica da madeira foi de $0,65 \mathrm{~g} . \mathrm{cm}^{-3}$ e da casca, igual a $0,49 \mathrm{~g} . \mathrm{cm}^{-3}$. Os estoques e os crescimentos em V, B, C e $\mathrm{CO}_{2}$ foram estimados nos dois estágios, Mata 1 e Mata 2. Pelo fato de as matas se encontrarem em estágios médio e avançado de regeneração, respectivamente, elas apresentavam estruturas, estoques e crescimentos distintos.
\end{abstract}

Palavras-chave: Floresta Atlântica, Estágio de sucessão e Sequestro de carbono.

\section{STOCKS AND GROWTHS OF VOLUME, BIOMASS, CARBON AND CARBON DIOXIDE IN SEASONAL SEMIDECIDUOUS FOREST}

\begin{abstract}
The objective of this work was to estimate the stock and the growth in volume (V), biomass (B), carbon $(\mathrm{C})$ and carbon dioxide $\left(\mathrm{CO}_{2}\right)$ in reserve areas of a Seasonal semideciduous forest in the Vale do Rio Doce, Minas Gerais State, Brazil. Data from the arboreous strata $(\mathrm{DBH} \geq 5.0 \mathrm{~cm})$ survey were used, and the permanent plots were measured in 2002 and 2007, in two native areas in a medium stage (Mata 1) and medium/ advanced stage (Mata2) of secondary regeneration. Based on data from the 2002 survey, species that showed the greatest percentages of volume and a minimum of five (5) individuals were selected to determine basic wood and bark densities of the trees. The average wood basic density was $0.65 \mathrm{~g} . \mathrm{cm}^{-3}$ and bark basic density

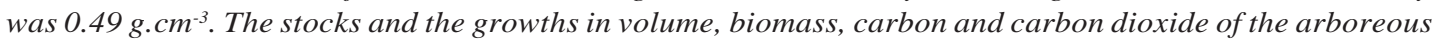
strata were estimated for Mata 1 and for Mata 2. Due to the fact that the forests are stage medium and advanced stages of regeneration respectively, they have different structures, stocks and growth.
\end{abstract}

Keywords: Atlantic Forest, Stage of regeneration and Carbon sequestration.

\section{INTRODUÇÃO}

Sequestro de carbono pelas florestas refere-se ao processo de absorção de dióxido de carbono $\left(\mathrm{CO}_{2}\right)$ do ar pelas plantas e fixação através da fotossíntese. Esse mecanismo de sequestrar e fixar o carbono atmosférico foi lançado na Convenção do Clima da
ONU como instrumento de flexibilização dos compromissos de diminuição das emissões de Gases de Efeito-Estufa (GEE) firmado pelos países (IPCC, 2000). Trata-se, portanto, de uma das modalidades dentro do Mecanismo de Desenvolvimento Limpo (MDL) do Protocolo de Quioto para compensar as emissões, no intuito de mitigar as mudanças climáticas.

\footnotetext{
${ }^{1}$ Recebido em 23.02.2010 e aceito para publicação em 31.10.2011.

${ }^{2}$ Departamento de Engenharia Florestal da Universidade Federal de Viçosa, UFV, Brasil. E-mail:<alsouza@ufv.br>, <csoares@ufv.br> e <bvital@ufv.br>.

${ }^{3}$ Programa de Pós-Graduação em Ciencia Florestal pela Universidade Federal de Viçosa, UFV, Brasil. E-mail:<alineboina@yahoo.com.br> e <ricogaspar.floresta@yahoo.com.br>

${ }^{4}$ Celulose Nipo Brasileira, CENIBRA, Brasil. E-mail: <jacinto.lana@cenibra.com.br>.
} 
As florestas desempenham papel primordial no ciclo do carbono: estocam mais carbono(C) nas árvores e no solo do que o existente na atmosfera (IPCC, 2000); emitem $C$ se perturbadas por causas antrópicas ou naturais; convertem-se em sumidouros mediante os processos de crescimento, produção e regeneração. Com informações sobre a taxa de mudança da biomassa e as razões que levaram essas mudanças a acontecerem, seria possível num futuro próximo realizar inferências sobre os estoques de carbono, suas fontes e sumidouros (HOUGHTON, 2005).

Estudo realizado pelo MCT (2008), entre 1988 e 1994, apontou que os valores líquidos de emissões causadas pelo desflorestamento bruto e remoções por regeneração por bioma foram, respectivamente: 151,7 TgC.ano ${ }^{-1}$ e 34,9 TgC.ano ${ }^{-1}$ (1 Tg corresponde a $10^{6}$ t) para o bioma Amazônia; 67,1 TgC.ano ${ }^{-1}$ e 15,7 TgC.ano ${ }^{-1}$ para o bioma Cerrado; 10,3 TgC.ano Tg $^{-1}$ e 2,8 TgC.ano-1 para o bioma Pantanal; 11,8 TgC.ano ${ }^{-1}$ e 0,5 TgC.ano-1 para o bioma Mata Atlântica; e 10,0 TgC.ano-1 de emissão para o bioma Caatinga.

Mesmo que já existam estimativas do estoque de carbono em diferentes biomas, estudos devem, no entanto, ser realizados com o objetivo de conhecer o potencial de estocagem, bem como os mecanismos (dinâmica) com que isso se processa. Como exemplo, os resultados de monitoramentos de parcelas permanentes instaladas em áreas de reservas protegidas em processos de recuperação ambiental inseridas nas propriedades rurais e nos projetos de base florestal e agropecuário podem contribuir muito para os estudos de dinâmica de $\mathrm{CO}_{2}$ nos ecossistemas de florestas naturais.

Assim, o objetivo deste estudo foi analisar a dinâmica dos estoques em volume, biomassa, carbono e dióxido de carbono em áreas de reservas protegidas em processos de recuperação ambiental inserida num projeto de base florestal.

\section{MATERIAL E MÉTODOS}

As áreas de estudo, doravante denominadas Mata 1 e Mata 2, localizam-se respectivamente a $19^{\circ} 30^{\prime}$

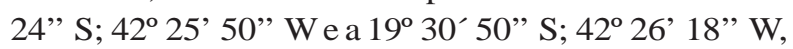
as quais se situam muito próximas e às margens da Lagoa Silvana, nos municípios de Caratinga e Bom Jesus do Galho, Vale do Rio Doce, MG. A tipologia vegetal original insere-se na região fitoecológica da Floresta Estacional Semidecidual Submontana e inclui-se no domínio da Floresta Atlântica. O clima predominante, pela classificação climática de Köppen, é o Aw - clima tropical úmido, com inverno seco e verão chuvoso. A temperatura máxima média é de $32,2{ }^{\circ} \mathrm{C}$ e a mínima média, de $19,4^{\circ} \mathrm{C}$. A umidade relativa média é de 59,8\%, a precipitação pluviométrica média mensal é de 106,6 mm e a evapotranspiração potencial anual varia entre 950 e $1.200 \mathrm{~mm}$.

As áreas pertencem à empresa Celulose Nipo Brasileira S.A. - CENIBRA e tem 302 ha de mata nativa, os quais estão preservados há aproximadamente 20 anos, sendo divididos em duas partes: uma com área de 272 ha (Mata 1) e a outra com 30 ha (Mata 2).

A dinâmica de sucessão das áreas de estudo está sendo monitorada por meio de parcelas permanentes de $500 \mathrm{~m}^{2}$ cada, instaladas em 2002 e inventariadas em 2002 e 2007. Foram lançadas 16 parcelas permanentes na Mata 1 e seis na Mata 2, perfazendo $8.000 \mathrm{~m}^{2}$ e $3.000 \mathrm{~m}^{2}$ de área amostral, respectivamente.

Cada árvore do estrato arbóreo (DAP $\geq 5,0 \mathrm{~cm}$ ) foi localizada dentro da parcela com suas coordenadas x e y e identificada, quando possível, em nível de espécie, tendo seu DAP e alturas total (Ht) e comercial (HC) mensurados. Para a amostragem do tronco e da casca e determinação das densidades, foram selecionadas as espécies que apresentavam, na época do inventário de 2002, maiores percentuais em volume e no mínimo cinco indivíduos.

Em cada espécie selecionada foram escolhidos aleatoriamente, nas parcelas de inventário, cinco indivíduos para coletas de madeira por meio de trado e casca por meio de formão. Essa amostragem contemplou apenas indivíduos do estrato arbóreo (DAP $\geq 5,0 \mathrm{~cm}$ ).

Os dados do inventário das parcelas permanentes realizado em 2007 foram analisados para a obtenção do índice de diversidade de Shannon-Weaver $\left(\mathrm{H}^{\prime}\right)$ e dos parâmetros estruturais: densidades; dominâncias, frequências; valores de cobertura e de importância; volumes; e distribuição dos diâmetros.

Os volumes totais de fuste e copa, com e sem casca, por espécie foram estimados mediante o emprego de equações de volumes de árvores individuais ajustadas para a mata secundária (SOARES et. al., 2011), sendo os volumes de casca do fuste, de galho com casca e de casca dos galhos obtidos pelas

Revista Árvore, Viçosa-MG, v.35, n.6, p.1277-1285, 2011 
seguintes expressões:

$\mathrm{vfC}_{i j}=\mathrm{vfCC}_{\mathrm{ij}}-\mathrm{vfsc}_{\mathrm{ij}}$; $\operatorname{vgcc}_{\mathrm{ij}}=\mathrm{vtcC}_{\mathrm{ij}}-\mathrm{vfCC}_{\mathrm{ij}}$; $\operatorname{vgc}_{\mathrm{ij}}=\operatorname{vgCC}_{\mathrm{ij}} \cdot \mathrm{F}_{\text {casca }}$

Fcasca $=\sum_{\mathrm{i}=1}^{\mathrm{n}} \mathrm{vfc}_{\mathrm{ij}} / \sum_{\mathrm{i}=1}^{\mathrm{n}} \mathrm{vfcc}_{\mathrm{ij}}$

em que:

$\mathrm{vfc}_{\mathrm{ij}}=$ volume de casca do fuste da $j$-ésima árvore da i-ésima espécie, em $\mathrm{m}^{3} ; \mathrm{vfcc}_{\mathrm{ij}}=$ volume do fuste com casca da $j$-ésima árvore da i-ésima espécie, em $\mathrm{m}^{3} ; \mathrm{vfsc}_{\mathrm{ij}}=$ volume do fuste sem casca da $j$-ésima árvore da i-ésima espécie, em $\mathrm{m}^{3}$; vgcc $_{\mathrm{ij}}=$ volume de galho com casca da $j$-ésima árvore da i-ésima espécie, em $\mathrm{m}^{3} ; \mathrm{vtcc}_{\mathrm{ij}}=$ volume total com casca da $j$-ésima árvore da i-ésima espécie, em m ${ }^{3}$; $\operatorname{vgc}_{\mathrm{ij}}=$ volume de casca do galho da $j$-ésima árvore da i-ésima espécie, em $\mathrm{m}^{3}$; e $\mathrm{F}_{\text {casca }}=$ fator de casca.

Os volumes por hectare e por espécie foram estimados pelo emprego das fórmulas a seguir:

$$
\begin{aligned}
& V F c c_{i}=\left(\sum_{j=1}^{n_{i}} v f c c_{i j}\right) / A_{k} ; V F s c_{i j}=\left(\sum_{j=1}^{n_{j}} v f s c_{i j}\right) / A_{k} ; V F C_{i}= \\
& \left(\sum_{j=1}^{n_{i}} v c_{i j}\right) / A_{k} ; V T c c_{i}=\left(\sum_{j=1}^{n_{i}} v t c c_{i j}\right) / A_{k} \\
& V G c c_{i}=\left(\sum_{j=1}^{n_{i}} v g c c_{i j}\right) / A_{k} ; V G c_{i}=\left(\sum_{j=1}^{n_{i}} v g c_{i j}\right) / A_{k} ; V G s c_{i}= \\
& V G c c_{i}-V G c_{i}
\end{aligned}
$$

em que:

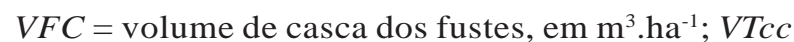
= volume total com casca, em $\mathrm{m}^{3} \cdot \mathrm{ha}^{-1} ; \mathrm{VFCC}=$ volume dos fustes com casca, em $\mathrm{m}^{3} \cdot \mathrm{ha}^{-1}$; VGCC = volume dos galhos com casca, em $\mathrm{m}^{3}$.ha- ${ }^{-1}$; $V G c=$ volume de casca dos galhos, em $\mathrm{m}^{3} \cdot \mathrm{ha}^{-1} ; \mathrm{A}_{\mathrm{k}}=$ área da amostragem de 0,80 ha para a mata 1 e 0,30 ha para a mata 2 , respectivamente.

Os estoques de biomassa e carbono e o sequestro de dióxido de carbono, em t.ha-1, em 2007, por espécie coletada, foram calculados pelo emprego das seguintes expressões:

$$
\begin{aligned}
& \text { BFuste }=D B m V F s c+B D B c V F . c ; B G a l h o= \\
& (V G c c-V G c)+D B c . V G c ;
\end{aligned}
$$

BTotal $=$ BFuste + Bgalho $;$ CTotal $=0,50$. BTotal $;$ e $\mathrm{CO}_{2}$ Total $=3,67$.CTotal; em que:

$\mathrm{DBm}$ = densidade básica da madeira, em t.m $\mathrm{m}^{-3}$; $\mathrm{DBc}=$ densidade básica da casca, em $\mathrm{t} . \mathrm{m}^{-3} ; \mathrm{VGcc}=$ volume por hectare dos galhos com casca, $\mathrm{em} \mathrm{m}^{3} \cdot \mathrm{ha}^{-1} ; \mathrm{VGc}=$ volume por hectare de casca dos galhos, em $\mathrm{m}^{3} \cdot \mathrm{ha}^{-1}$; BFuste $=$ estoque de biomassa de fuste, em t.ha ${ }^{-1}$; BGalho = biomassa de fuste, em t.ha-1 ${ }^{-1}$ BTotal = biomassa total, em t.ha-1;

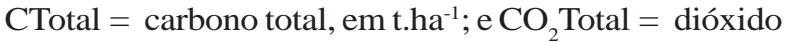
de carbono total, em t.ha ${ }^{-1}$; VFsc $=$ volume por hectare do fuste sem casca, $\mathrm{em} \mathrm{m}^{3} \cdot \mathrm{ha}^{-1} ; \mathrm{e} \mathrm{VFc}=$ volume por hectare de casca dos fustes, em $\mathrm{m}^{3} \cdot \mathrm{ha}^{-1}$.

Para calcular os estoques de biomassa das espécies não coletadas, consideraram-se as médias das densidades básicas da madeira e da casca iguais às médias das densidades básicas da madeira e da casca das espécies coletadas.

O fator de conversão de estoque de carbono em dióxido de carbono igual a 3,67 foi obtido pela razão entre a massa molecular do dióxido $\left(\mathrm{CO}_{2}\right)$ igual a 44 e a massa atômica do carbono (C) igual a 12 (HOEN; SOLBERG, 1994).

Finalmente, os estoques de volume, biomassa e carbono e o sequestro de dióxido de carbono das espécies coletadas e das não coletadas foram totalizados para os seguintes compartimentos: fuste com casca (FCc), fuste sem casca (Fsc) e total com casca (Tcc).

\section{RESULTADOS}

Na área em estágio médio de regeneração (Mata 1), em 2007, foram identificadas 117 espécies arbóreas e nove indeterminadas pertencentes a 83 gêneros de 42 famílias botânicas, com índice de diversidade de Shannon Weaver (H') igual a 3,49, densidade total (DTA) de 1.459 indivíduos/ha (n.ha-1), dominância total de $16,82 \mathrm{~m}^{2}$.ha-1 e volume total com casca igual a 115,40 m³ $\mathrm{ha}^{-1}$. Na área em estágio avançado de regeneração (Mata 2), foram identificadas 86 espécies arbóreas e seis indeterminadas pertencentes a 72 gêneros de 38 famílias botânicas, com índice de diversidade de Shannon Weaver (H') igual a 3,79, densidade total (DTA) de 1.897 n.ha-1, dominância total de $25,73 \mathrm{~m}^{2}$.ha $\mathrm{h}^{-1}$ e volume total com casca igual a $245,20 \mathrm{~m}^{3} \cdot \mathrm{ha}^{-1}$.

Na área em estágio médio de regeneração (Mata 1), foram selecionadas 34 espécies (25\%) para as coletas de madeira e de casca, as quais apresentaram 1.390n.ha ${ }^{-1}$ (95\%), 11,63 m².ha-1 (69\%), 92,11 m³.ha' (80\%)\% do volume total com casca, $75 \%$ do valor de cobertura

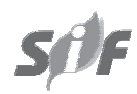

Revista Árvore, Viçosa-MG, v.35, n.6, p.1277-1285, 2011 
(VC) e 71\% do valor de importância fitossociológico (VI). As demais espécies (75\%), denominadas não coletadas, contribuíram com 23,29 $\mathrm{m}^{3} \cdot \mathrm{ha}^{-1}$ (20\%).

$\mathrm{Na}$ área em estágio avançado de regeneração (Mata 2), foram selecionadas 16 espécies (16\%) para coletas de madeira e de casca, que representaram 1.146 n.ha-1 $(60 \%), 18,40 \mathrm{~m}^{2} . \mathrm{ha}^{-1}$ (71\%), 202,40 $\mathrm{m}^{3} \cdot \mathrm{ha}^{-1}$ (82\%) do volume total com casca, 67\% de VC e 56\% de VI. As demais espécies (84\%), denominadas não coletadas, contribuíram com 42,80 $\mathrm{m}^{3} \cdot \mathrm{ha}^{-1}$ (18\%) do volume total com casca.

A média da densidade básica da madeira foi de 0,65 g.cm ${ }^{-3}$ e da casca, igual a 0,49 g.cm ${ }^{-3}$, cujas médias, mediante a aplicação do teste t para dados não pareados, a $95 \%$ de probabilidade, diferiam entre si (" $\mathrm{t}$ " calculado $=6,385 \mathrm{e}$ "t" tabelado $=2,045$ ).

Na área em estágio médio de regeneração (Tabela 1), verificou-se que os volumes totais incluindo madeira e casca dos troncos e galhos, em 272 ha, foram, respectivamente, de 29.368,90 $\mathrm{m}^{3}\left(107,97 \mathrm{~m}^{3} \cdot \mathrm{ha}^{-1}\right)$ e 31.390,54 m (115,41 $\mathrm{m}^{3}$.ha-1), em 2002 e 2007. Entre os anos 2002 e 2007 houve acréscimo de 7,43 m³ .ha $^{-1}$ de volume total com casca, isto é, crescimento médio anual de $1,49 \mathrm{~m}^{3} \cdot \mathrm{ha}^{-1}$. nno $^{-1}$. O estoque total de biomassa evoluiu de 19.514,17 tB (71,74 tB.ha-1) em 2002 para 19.879,71 tB (73,09 tB.ha $\left.{ }^{-1}\right)$ em 2007, tendo crescimento periódico médio anual de 0,27 tB.ha-1 ano $^{-1}$.

Tabela 1 - Estoque e crescimento em volume, biomassa, carbono e dióxido de carbono nos anos de 2002 e de 2007, por hectare e na_área total (272 ha) da Mata 1 (estágio médio de regeneração). Fcc = fuste com casca, Fsc = fuste sem casca e Tcc $=$ total com casca.

Table 1 -Stocks and growths in volume, biomass, carbon and carbon dioxide in the years 2002 and 2007, per hectare and per total area (272 ha) of Mata 1. In which: FCC = Stem including bark; Fsc = Stem excluding bark; Fcasca = bark of the Stem; Gcc = branch including bark; Gsc = branch excluding bark; Gcasca = bark of the branch; and TCC= total including bark.

\begin{tabular}{|c|c|c|c|c|c|c|c|c|}
\hline \multirow[t]{3}{*}{ Parâmetros } & \multicolumn{4}{|c|}{ Estoques de Volume } & \multicolumn{4}{|c|}{ Crescimento de Volume } \\
\hline & \multicolumn{2}{|c|}{2002} & \multicolumn{2}{|c|}{2007} & \multicolumn{2}{|c|}{ Entre 2002-2007 } & \multicolumn{2}{|c|}{ Por Ano } \\
\hline & $\overline{\mathrm{m}^{3} \mathrm{ha}^{-1}}$ & $\begin{array}{l}\mathrm{m}^{3} \text { em } \\
272 \mathrm{ha}\end{array}$ & $\mathrm{m}^{3} \mathrm{ha}^{-1}$ & $\begin{array}{l}\mathrm{m}^{3} \text { em } \\
272 \text { ha }\end{array}$ & $\mathrm{m}^{3} \mathrm{ha}^{-1}$ & $\begin{array}{l}\mathrm{m}^{3} \text { em } \\
272 \text { ha }\end{array}$ & $\mathrm{m}^{3}$ ha & $\begin{array}{l}\mathrm{m}^{3} \text { em } \\
272 \mathrm{ha}\end{array}$ \\
\hline Fcc & 81,81 & 22252,89 & 87,84 & 23891,64 & 6,02 & 1638,75 & 1,20 & 327,75 \\
\hline Fsc & 67,28 & 18299,83 & 71,89 & 19553,78 & 4,61 & 1253,95 & 0,92 & 250,79 \\
\hline Tcc & 107,97 & 29368,90 & 115,41 & 31390,54 & 7,43 & 2021,64 & 1,49 & 404,33 \\
\hline \multirow[t]{3}{*}{ Parâmetros } & \multicolumn{4}{|c|}{ Estoques de Biomassa } & \multicolumn{4}{|c|}{ Crescimento de Biomassa } \\
\hline & \multicolumn{2}{|c|}{2002} & \multicolumn{2}{|c|}{2007} & \multicolumn{2}{|c|}{ Entre 2002-2007 } & \multicolumn{2}{|c|}{ Por Ano } \\
\hline & $\mathrm{t} \mathrm{ha}^{-1}$ & $\begin{array}{c}t \text { em } \\
272 \text { ha } \\
\end{array}$ & $\mathrm{t} \mathrm{ha}^{-1}$ & $\begin{array}{c}\mathrm{t} \text { em } \\
272 \text { ha } \\
\end{array}$ & $\mathrm{t} \mathrm{ha}^{-1}$ & $\begin{array}{c}\mathrm{t} \text { em } \\
272 \text { ha } \\
\end{array}$ & $\mathrm{t} \mathrm{ha}^{-1}$ & $\begin{array}{c}\mathrm{t} \text { em } \\
272 \mathrm{ha} \\
\end{array}$ \\
\hline Fcc & 54,32 & 14775,90 & 55,67 & 15141,44 & 1,34 & 365,54 & 0,27 & 73,11 \\
\hline Fsc & 46,83 & 12737,01 & 47,76 & 12991,52 & 0,94 & 254,51 & 0,19 & 50,90 \\
\hline Tcc & 71,74 & 19514,17 & 73,09 & 19879,71 & 1,34 & 365,54 & 0,27 & 73,11 \\
\hline \multirow[t]{3}{*}{ Parâmetros } & \multicolumn{4}{|c|}{ Estoques de Carbono } & \multicolumn{4}{|c|}{ Crescimento de Carbono } \\
\hline & \multicolumn{2}{|c|}{2002} & \multicolumn{2}{|c|}{2007} & \multicolumn{2}{|c|}{ Entre 2002-2007 } & \multicolumn{2}{|c|}{ Por Ano } \\
\hline & $\mathrm{t} \mathrm{ha}^{-1}$ & $\begin{array}{c}\mathrm{t} \text { em } \\
272 \mathrm{ha} \\
\end{array}$ & $\mathrm{tha}^{-1}$ & $\begin{array}{c}\mathrm{t} \text { em } \\
272 \text { ha }\end{array}$ & $\mathrm{t} \mathrm{ha}^{-1}$ & $\begin{array}{c}\mathrm{t} \text { em } \\
272 \text { ha }\end{array}$ & $\mathrm{t} \mathrm{ha}^{-1}$ & $\begin{array}{c}\mathrm{t} \text { em } \\
272 \text { ha } \\
\end{array}$ \\
\hline Fcc & 27,14 & 7382,66 & 27,83 & 7570,72 & 0,69 & 188,06 & 0,14 & 37,61 \\
\hline Fsc & 23,40 & 6363,71 & 23,88 & 6495,76 & 0,49 & 132,05 & 0,10 & 26,41 \\
\hline Tcc & 35,87 & 9756,29 & 36,54 & 9939,85 & 0,67 & 183,56 & 0,13 & 36,71 \\
\hline \multirow[t]{3}{*}{ Parâmetros } & \multicolumn{4}{|c|}{ Seqüestro de Dióxido de Carbono } & \multicolumn{4}{|c|}{ Seqüestro de Dióxido de Carbono } \\
\hline & \multicolumn{2}{|c|}{2002} & \multicolumn{2}{|c|}{2007} & \multicolumn{2}{|c|}{ Entre 2002-2007 } & \multicolumn{2}{|c|}{ Por Ano } \\
\hline & $\mathrm{t} \mathrm{ha}^{-1}$ & $\begin{array}{c}t \text { em } \\
272 \text { ha }\end{array}$ & $\mathrm{t} \mathrm{ha}^{-1}$ & $\begin{array}{c}\mathrm{t} \text { em } \\
272 \text { ha }\end{array}$ & $\mathrm{t} \mathrm{ha}^{-1}$ & $\begin{array}{l}\text { t em } \\
272 \text { ha }\end{array}$ & $\mathrm{t} \mathrm{ha}^{-1}$ & $\begin{array}{c}\mathrm{t} \text { em } \\
272 \text { ha }\end{array}$ \\
\hline Fcc & 99,61 & 27094,35 & 102,15 & 27784,54 & 2,54 & 690,19 & 0,51 & 138,04 \\
\hline Fsc & 85,86 & 23354,82 & 87,65 & 23839,44 & 1,78 & 484,62 & 0,36 & 96,92 \\
\hline Tcc & 131,64 & 35805,60 & 134,11 & 36479,26 & 2,48 & 673,66 & 0,50 & 134,73 \\
\hline
\end{tabular}

Revista Árvore, Viçosa-MG, v.35, n.6, p.1277-1285, 2011 
Os estoques totais de carbono da Mata 1, em 2002 e 2007, foram, respectivamente, 9.756,29 tC (35,87 tC.ha-1) e 9.939,85 tC (36,54 tC.ha-1), ou seja, teve crescimento periódico médio anual de 0,13 tC.ha- ${ }^{-1}$.ano ${ }^{-1}$. Já os estoques de dióxido de carbono foram de 35.805,60 $\mathrm{tCO}_{2}$ $\left(131,64 \mathrm{tCO}_{2} \cdot \mathrm{ha}^{-1}\right)$ e $36.479,26 \mathrm{tCO}_{2}\left(134,11 \mathrm{tCO}_{2} \cdot \mathrm{ha}^{-1}\right)$, respectivamente, em 2002 e 2007 , tendo um sequestro periódico anual médio de $0,50 \mathrm{tCO}_{2} \cdot \mathrm{ha}^{-1} \cdot \mathrm{ano}^{-1}$.

Na área em estágio avançado (Tabela 2), verificou-se que os volumes totais incluindo madeira e casca dos troncos e galhos, em $30 \mathrm{ha}$, foram de 6.789,18 m $\left(226,31 \mathrm{~m}^{3} \cdot \mathrm{ha}^{-1}\right)$ e 7.356,03 m $\left(245,20 \mathrm{~m}^{3} \cdot \mathrm{ha}^{-1}\right)$, respectivamente, em 2002 e 2007, tendo incremento periódico anual (IPA) de $3,78 \mathrm{~m}^{3} \cdot \mathrm{ha}^{-1}$. $\mathrm{ano}^{-1}$. Os estoques de biomassa total com casca foram de 146,84 tB.ha ${ }^{-1}$

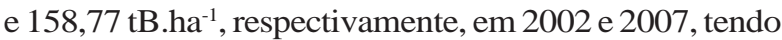
IPA de 2,39 tB.ha-1 ano $^{-1}$. Os estoques de carbono foram de 2.202,57 tC (73,42 tC.ha-1) em 2002 e de 2.381,52 tC (79,38 tC.ha-1) em 2007, com IPA de 1,19 tC.ha-1. ano $^{-1}$. Já o sequestro de dióxido de carbono totalizou 9.083,44 tCO $\left(269,45 \mathrm{tCO}_{2}\right.$. ha-1) e 8.740,18 $\mathrm{tCO}_{2}\left(291,34 \mathrm{tCO}_{2} \cdot \mathrm{ha}^{-1}\right)$, respectivamente, em 2002 e 2007, com IPA de 4,38 tCO 2 . ha $a^{-1}$. ano $^{-1}$.

As distribuições diamétricas das Matas 1 e 2 apresentaram tendência de “J-invertido” típica de florestas naturais inequiâneas (Figuras 1A e 2A). Na Mata 1, cerca de 95\% dos estoques de volume e biomassa e sequestro de $\mathrm{CO}_{2}$ estavam concentrados nas árvores

Tabela 2 - Estoques e crescimentos em volume (V), biomassa (B), carbono (C) e dióxido de carbono $\left(\mathrm{CO}_{2}\right)$ nos anos de 2002 e de 2007, por hectare e na área total (30 ha) da Mata 2 (estágio avançado de regeneração). Fcc = fuste com casca, Fsc = fuste sem casca e Tcc = total com casca.

Table 2 - Stocks and growths in volume, biomass, carbon and carbon dioxide in the years 2002 and 2007, per hectare and per total area (30 ha) of Mata 2. In which: FCC = Stem including bark; Fsc = Stem excluding bark; Fcasca = bark of the Stem; Gcc = branch including bark; Gsc = branch excluding bark; Gcasca = bark of the branch; and TCC = total including bark.

\begin{tabular}{|c|c|c|c|c|c|c|c|c|}
\hline \multirow[t]{3}{*}{ Parâmetros } & \multicolumn{4}{|c|}{ Estoques de Volume } & \multicolumn{4}{|c|}{ Crescimento de Volume } \\
\hline & \multicolumn{2}{|c|}{2002} & \multicolumn{2}{|c|}{2007} & \multicolumn{2}{|c|}{ Entre 2002-2007 } & \multicolumn{2}{|c|}{ Por Ano } \\
\hline & $\overline{\mathrm{m}^{3} \mathrm{ha}^{-1}}$ & $\begin{array}{l}\mathrm{m}^{3} \text { em } \\
30 \mathrm{ha}\end{array}$ & $\mathrm{m}^{3} \mathrm{ha}^{-1}$ & $\begin{array}{l}\mathrm{m}^{3} \text { em } \\
30 \mathrm{ha}\end{array}$ & $\mathrm{m}^{3} \mathrm{ha}^{-1}$ & $\begin{array}{l}\mathrm{m}^{3} \text { em } \\
30 \text { ha }\end{array}$ & $\mathrm{m}^{3} \mathrm{ha}^{-1}$ & $\begin{array}{l}\mathrm{m}^{3} \mathrm{em} \\
30 \mathrm{ha} \\
\end{array}$ \\
\hline Fcc & 183,15 & 5494,54 & 197,77 & 5933,12 & 14,62 & 438,59 & 2,92 & 87,72 \\
\hline Fsc & 155,84 & 4675,17 & 167,38 & 5021,50 & 11,54 & 346,33 & 2,31 & 69,27 \\
\hline Tcc & 226,31 & 6789,18 & 245,20 & 7356,03 & 18,90 & 566,85 & 3,78 & 113,37 \\
\hline \multirow[t]{3}{*}{ Parâmetros } & \multicolumn{4}{|c|}{ Estoques de Biomassa } & \multicolumn{4}{|c|}{ Crescimento de Biomassa } \\
\hline & \multicolumn{2}{|c|}{2002} & \multicolumn{2}{|c|}{2007} & \multicolumn{2}{|c|}{ Entre 2002-2007 } & \multicolumn{2}{|c|}{ Por Ano } \\
\hline & $\mathrm{t} \mathrm{ha}^{-1}$ & $\begin{array}{l}\mathrm{t} \text { em } \\
30 \text { ha }\end{array}$ & $\mathrm{t} \mathrm{ha}^{-1}$ & $\begin{array}{l}\mathrm{t} \text { em } \\
30 \text { ha }\end{array}$ & $\mathrm{t} \mathrm{ha}^{-1}$ & $\begin{array}{l}\mathrm{t} \text { em } \\
30 \text { ha }\end{array}$ & $\mathrm{t} \mathrm{ha}^{-1}$ & $\begin{array}{l}\mathrm{t} \text { em } \\
30 \mathrm{ha} \\
\end{array}$ \\
\hline Fcc & 118,41 & 3552,29 & 127,61957 & 3828,59 & 9,21 & 276,29 & 1,84 & 55,26 \\
\hline Fsc & 100,72 & 3021,72 & 107,99262 & 3239,78 & 7,27 & 218,05 & 1,45 & 43,61 \\
\hline Tcc & 146,84 & 4405,15 & 158,76796 & 4763,04 & 11,93 & 357,89 & 2,39 & 71,58 \\
\hline \multirow[t]{3}{*}{ Parâmetros } & \multicolumn{4}{|c|}{ Estoques de Carbono } & \multicolumn{4}{|c|}{ Crescimento de Carbono } \\
\hline & \multicolumn{2}{|c|}{2002} & \multicolumn{2}{|c|}{2007} & \multicolumn{2}{|c|}{ Entre 2002-2007 } & \multicolumn{2}{|c|}{ Por Ano } \\
\hline & $\mathrm{t} \mathrm{ha}^{-1}$ & $\begin{array}{c}\mathrm{t} \text { em } \\
30 \text { ha }\end{array}$ & $\mathrm{t} \mathrm{ha}^{-1}$ & $\begin{array}{c}\mathrm{t} \text { em } \\
30 \text { ha }\end{array}$ & $\mathrm{t} \mathrm{ha}^{-1}$ & $\begin{array}{c}t \text { em } \\
30 \text { ha }\end{array}$ & $\mathrm{t} \mathrm{ha}^{-1}$ & $\begin{array}{c}\mathrm{t} \text { em } \\
30 \mathrm{ha} \\
\end{array}$ \\
\hline Fcc & 59,20 & 1776,15 & 63,81 & 1914,29 & 4,60 & 138,15 & 0,92 & 27,63 \\
\hline Fsc & 50,36 & 1510,86 & 54,00 & 1619,89 & 3,63 & 109,03 & 0,73 & 21,81 \\
\hline Tcc & 73,42 & 2202,57 & 79,38 & 2381,52 & 5,96 & 178,95 & 1,19 & 35,79 \\
\hline \multirow[t]{3}{*}{ Parâmetros } & \multicolumn{4}{|c|}{ Seqüestro de Dióxido de Carbono } & \multicolumn{4}{|c|}{ Seqüestro de Dióxido de Carbono } \\
\hline & \multicolumn{2}{|c|}{2002} & \multicolumn{2}{|c|}{2007} & \multicolumn{2}{|c|}{ Entre 2002-2007 } & \multicolumn{2}{|c|}{ Por Ano } \\
\hline & $\mathrm{t} \mathrm{ha}^{-1}$ & $\begin{array}{c}\mathrm{t} \text { em } \\
272 \text { ha }\end{array}$ & $\mathrm{t} \mathrm{ha}^{-1}$ & $\begin{array}{c}\mathrm{t} \text { em } \\
272 \text { ha }\end{array}$ & $\mathrm{t} \mathrm{ha}^{-1}$ & $\begin{array}{l}\mathrm{t} \text { em } \\
272 \text { ha }\end{array}$ & $\mathrm{t} \mathrm{ha}^{-1}$ & $\begin{array}{c}\mathrm{t} \text { em } \\
272 \mathrm{ha} \\
\end{array}$ \\
\hline Fcc & 217,28 & 6518,46 & 234,18 & 7025,46 & 16,90 & 507,00 & 3,38 & 101,40 \\
\hline Fsc & 184,83 & 5544,86 & 198,17 & 5944,99 & 13,34 & 400,13 & 2,67 & 80,03 \\
\hline Tcc & 269,45 & 8083,44 & 291,34 & 8740,18 & 21,89 & 656,73 & 4,38 & 131,35 \\
\hline
\end{tabular}


com até 45 cm de DAP (Figuras 1B e 1C); e na Mata 2, mais de $35 \%$ estavam estocados nas árvores com DAP $\geq 45 \mathrm{~cm}$ (Figura 2B e 2C), indicando que a Mata 2 se encontrava em estágio de sucessão mais avançado.

Na área em estágio médio de regeneração, os fustes com casca contribuíram com $76 \%$ do estoque da biomassa total com casca, seguido pelos galhos com casca com $24 \%$. Discriminando separadamente a madeira e a casca,
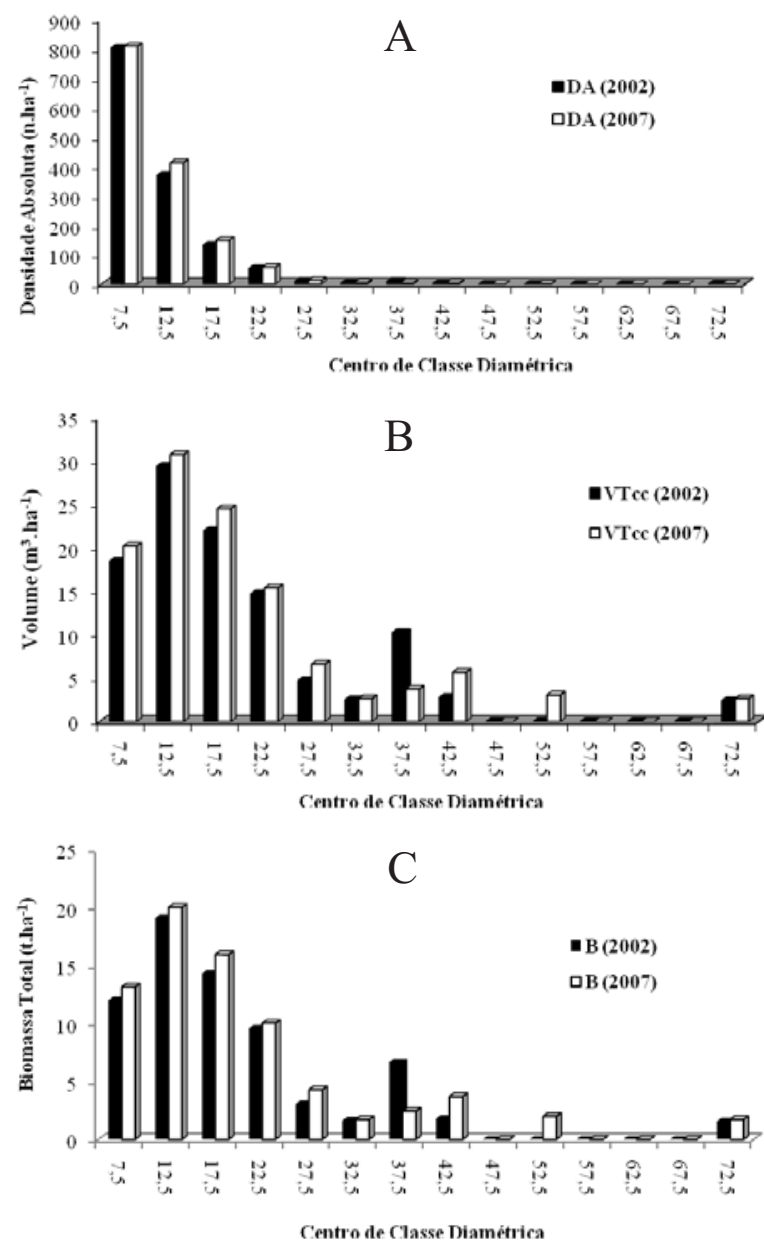

Figura 1 - Distribuição por classe diamétrica do número de indivíduos (DA) por hectare (A) e dos estoques de volume total com casca (VTcc), em $\mathrm{m}^{3} \cdot \mathrm{ha}^{-1}$ (B); e biomassa total com casca (B), em t.ha-1 (C), da Mata 1 nos anos de 2002 e 2007.

Figure 1 - Distribution per center of diameter class of the number of individuals (DA) per hectare (A), of the stocks of total including bark volume (VTCC), in $\mathrm{m}^{3} \cdot \mathrm{ha}^{-1}(\mathrm{~B})$, and biomass including bark (B), in ton.ha ${ }^{-1}(C)$, of Mata 1 in the years 2002 and 2007. em ordem decrescente de contribuição na estocagem e no sequestro, os fustes sem casca (FSC) contribuíram com $65 \%$, os galhos sem casca com $20 \%$, a casca dos fustes (Fcasca) com 11\% e a casca dos galhos (Gcasca) com 3\%.

Na área em estágio avançado de regeneração, verificou-se que os fustes com casca contribuíram com $81 \%$ e os galhos com casca, com $19 \%$. Em ordem
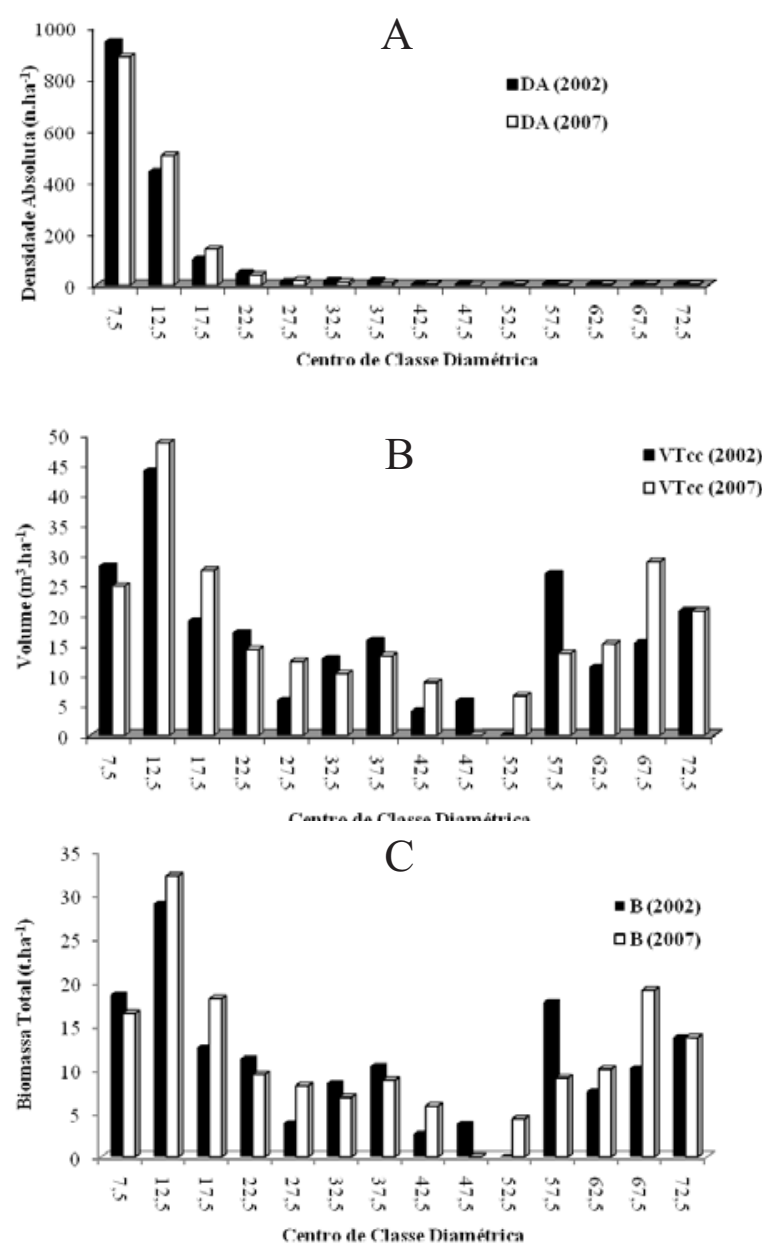

Figura 2 - Distribuição por classe diamétrica do número de indivíduos (DA) por hectare (A) e dos estoques de volume total com casca (VTcc), em $\mathrm{m}^{3} \cdot \mathrm{ha}^{-1}$ (B); e biomassa total com casca (B), em t.ha ${ }^{-1}$ (C), da Mata 2 nos anos de 2002 e 2007.

Figure 2 - Distribution per diameter class of the number of individuals (DA) per hectare(A), of the stocks of total including bark volume (VTcC), in $\mathrm{m}^{3} \cdot \mathrm{ha}^{-1}(\mathrm{~B})$, and biomass including bark (B), in ton. ha ${ }^{-1}(C)$, of Mata 2 in the years 2002 and 2007. 
decrescente de contribuição, os componentes foram: fustes sem casca (72\%), galhos sem casca (16\%), casca dos fustes (10\%) e casca dos galhos (3\%).

Comparando as duas áreas, observou-se que a área em estágio avançado de regeneração apresentou maior acúmulo de biomassa total que a área em estágio médio e que a biomassa do tronco aumentou e a de galho diminuiu, confirmando a influência dos estágios evolutivos.

\section{DISCUSSÃO}

Comparativamente aos estudos realizados por Drumond et al. (1996) na região do Vale do Rio Doce, nas proximidades do Parque Florestal Estadual do Rio Doce-PFERD, em Minas Gerais, as Matas 1 e 2 diferiram, respectivamente, da Mata Mombaça, em estágio médio de regeneração, com $H^{\prime}$ igual a 3,09, 1.247 n.ha-1, 16,70 m². ha $^{-1}$ e 205,3 m³ ha $^{-1}$ e da Mata Salão Dourado, em estágio avançado de regeneração, com $\mathrm{H}^{\prime}$ igual a 3,85, 1.690 n.ha-1 $30,56 \mathrm{~m}^{2} \cdot$ ha $^{-1}$ e 381,76 m³ ha $^{-1}$.

Os estoques de biomassa total com casca nos anos de 2002 e 2007, no estágio médio de regeneração (Tabela 1), foram inferiores ao valor encontrado por Drumond et al. (1997) em Floresta Estacional Semidecidual, Vale

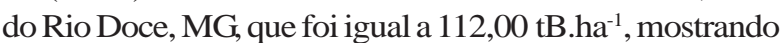
a elevada variabilidade entre as áreas na região deste estudo. Em 2007, a biomassa de fustes com casca foi inferior e a de galhos com casca $(17,42$ tB.ha-1), superior aos valores obtidos por Drumond et al. (1997), que estimaram os estoques de biomassa de tronco em 93,3 tB.ha ${ }^{-1}$ e de galhos em 14,3 tB.ha-1.

Segundo Santos et al. (2004), entretanto, em trabalho apresentado sobre estimativa da biomassa seca acima do solo e estoque de carbono de sistemas agroflorestais (SAF) estudados nas várzeas do rio Juba, município de Cametá, Estado do Pará, a biomassa seca dos SAFs foi de 298,44 tB.ha-1. Sierra et al. (2007), estudando estoque de carbono total em florestas tropicais na Colômbia, encontraram, em floresta primária, uma média de 383,7 $228 \pm 55,5$ tC.ha-1 e, em florestas secundárias, a média de 228,2 \pm 13,1 228 tC. ha ${ }^{-1}$. A biomassa média acima do solo presente nas florestas primárias na Amazônia brasileira, segundo Fearnside (2002), é de 354 tB.ha-1, dos quais 28 tB.ha-1 dessa biomassa está morta.

Em ambas as áreas, o tronco foi o compartimento que mais contribuiu com a biomassa total, cujo resultado foi semelhante aos de Soares e Oliveira (2002) e
Paixão et al. (2006) em plantios de eucalipto. Drumond et al. (1997) verificaram a seguinte distribuição da biomassa nos componentes arbóreos: tronco (77\%), galhos (13\%) e folha (4\%).

Em um estudo realizado por Watzlawick et al. (2002), os diferentes compartimentos tiveram participações diferentes, dependendo do estágio de sucessão: no estágio inicial, o maior percentual foi de raízes (27\%), seguidas da madeira (23\%), dos galhos vivos (20\%) e do subdossel (17\%); e no estágio médio, o maior percentual foi de madeira (30\%), seguida dos galhos vivos (26\%) e da casca (9\%). À medida que evolui o estágio de sucessão, aumenta os estoques de volume, biomassa e carbono. Contudo, as contribuições dos estoques por compartimento podem diferir muito entre comunidades em diferentes estágios de sucessão, dependendo da composição de espécies arbóreas e da distribuição diamétrica das comunidades.

Glenday (2006) destacou que os projetos de base florestal são importantes tanto para mitigar as mudanças climáticas quanto para promover o manejo florestal sustentável. Em estudo conduzido na Floresta Nacional de Kakamega, essa autora encontrou na área indígena estoque de $330 \pm 65128$ tC.ha $^{-1}$.

Em estudo realizado em florestas submontanas no Sudeste asiático, Hertel et al. (2009) encontraram um estoque de 303 tB.ha-1, equivalente a 128 tC.ha-1, e os componentes acima do solo corresponderam a 286 tB.ha-1, enquanto raízes grossas e finas corresponderam a 11,2 tB.ha-1 e 5,6 tB.ha-1, respectivamente.

Higuchi et al. (2004), estudando a dinâmica e balanço de carbono em vegetação primária na Amazônia central, encontram, em 12 anos de monitoramento, incremento de carbono de 1,2 t.ha-1 .ano $^{-1}$. Ressaltaram que no período estudado a região foi afetada pelos fenômenos climáticos El Niño (período de seca anormal) e La Niña (período de chuva anormal).

Melo e Duringan (2006) compararam a fixação de carbono em reflorestamentos de matas ciliares na região do Vale do Paranapanema, Estado de São Paulo, e verificaram que o estoque de carbono nas matas ciliares reflorestadas em área de cerrado foi de 50 tC.ha-1 e em solos com cobertura florestal, de 79,7 tC.ha-1.

Tiepolo et al. (2002), em estudo de medição e monitoramento do estoque de carbono no Projeto de Ação Climática de Guaraqueçaba, Estado do Paraná, 
observaram que o estoque médio de carbono acima do solo foi de 135,9 tC. ha ${ }^{-1}$ na floresta submontana, 106,8 tC. ha $^{-1}$ na floresta de planície, 64,12 tC.ha- ${ }^{-1}$ na floresta de várzea, 106,1 tC.ha ${ }^{-1}$ na floresta no estágio avançado, 101,96 tC.ha- ${ }^{-1}$ na floresta secundária em estágio médio e 42,89 tC.ha-1 na floresta secundária jovem. Seus estudos ainda contemplaram áreas de pastagem com 2,4 tC.ha-1 e áreas de moitas com 7,4 tC.ha-1 .

Vogel et al. (2006) quantificaram a produção total de biomassa cima do solo de uma Floresta Estacional Decidual, em Itaara, RS, estimando-a em 210 t.ha-1. Verificaram que os galhos contribuíram com $48 \%$, a madeira do fuste com $43,3 \%$, a casca do fuste com $5,4 \%$ e as folhas com $2,4 \%$.

Luyssaert et al. (2008), compilando resultados de artigos publicados sobre as florestas maduras como sumidouros globais de carbono, encontraram informações que os levaram a concluir que a produtividade líquida de carbono, incluindo o carbono do solo, era geralmente positiva, corroborando a hipótese de que as florestas maduras continuam a acumular carbono, contrapondo a hipótese de que essas florestas apresentam fluxo neutro desse elemento.

Nascimento et al. (2002) estimaram em 397,7 \pm 30 t.ha-1 a biomassa acima do solo na Amazônia central (80 km de Manaus). A participação dos componentes foi a seguinte: árvores com DAP $\geq 10 \mathrm{~cm}$ representaram $81,9 \%$ da biomassa total, resíduos lenhosos 7\%, pequenas árvores, arbustos e plântulas com DAP < 10 cm 5,3\% e lianas, litter, fuste de pequenas palmeiras 2,1\%, 1,9\%, 0,3 , respectivamente.

\section{CONCLUSÕES}

Com base nos resultados apresentados e discutidos, chegou-se às seguintes conclusões:

1. A floresta nativa em estágio avançado de regeneração (Mata 2) estocou mais que o dobro em volume e biomassa e sequestrou aproximadamente nove vezes mais carbono e dióxido de carbono em comparação com a Mata 1.

2. Na área em estágio médio (Mata 1), cerca de $75 \%$ dos estoques de volume e biomassa e sequestro de $\mathrm{CO}_{2}$ estão concentrados nas árvores com até $45 \mathrm{~cm}$ de DAP, e na área em estágio avançado (Mata 2) mais de 35\% estão estocados nas árvores com $\mathrm{DAP} \geq 45 \mathrm{~cm}$.
3. Em ordem decrescente de contribuição na estocagem de biomassa e carbono e sequestro de $\mathrm{CO}_{2}$ na Mata 1, os fustes sem casca contribuíram com 65\%, seguidos dos galhos com casca com $24 \%$ e da casca dos fustes com 11\%. Na Mata 2, os fustes sem casca participaram com $68 \%$, os galhos com casca com $20 \%$ e a casca dos fustes com $12 \%$.

4. As diferenças observadas nos estoques de volume, biomassa e carbono e no sequestro de $\mathrm{CO}_{2}$ no componente arbóreo podem ser atribuídas à composição florística, à estrutura diamétrica e ao estágio de sucessão.

\section{REFERÊNCIAS}

BRASIL. Ministério da Ciência e Tecnologia.

Emissões e remoções de dióxido de carbono devidas a mudanças nos estoques de florestas plantadas. Primeiro Inventário Brasileiro de Emissões Antrópicas de Gases de Efeito Estufa - Relatórios de Referência. Disponível em: <http:// www.mct.gov.br/index.php/content/ /view/ 21456.html >. Acesso em: 23 jan. 2008.

DRUMOND, M. A. et al. Distribuição de biomassa e de nutrientes em diferentes coberturas florestais e pastagens na região do Médio Rio Doce-MG. Revista Árvore, v.21, n.2, p.187-199, 1997.

DRUMOND, M. A. et al. Alterações fitossociológicas e edáficas na Mata Atlântica em função das modificações da cobertura vegetal. Revista Árvore, v.20, n.4, p.461-466, 1996.

FEARNSIDE, P. M. Fogo e emissão de gases de efeito estufa dos ecossistemas florestais da Amazônia brasileira. Estudos Avançados, v.16, n.44, p.99-123, 2002.

GLENDAY, J. Carbon storage and emissions offset potential in an East African tropical rainforest. Forest Ecology and Management, v.235, n.1, p.72-83, 2006.

HERTEL, D. et al. Below- and above-ground biomass and net primary production in a paleotropical natural forest (Sulawesi, Indonesia) as compared to neotropical forests. Forest Ecology and Management, v.258, n.9, p.1904-1912, 2009. 
HIGUCHI, N. et al. Dinâmica e balanço do carbono da vegetação primária da Amazônia Central. Revista Floresta, v.34, n.3, p.295-304. 2004.

HOEN, H.; SOLBERG, B. Potential and economic efficiency of carbon sequestration in Forest biomass through silvicultural management. Forest Science, v.40, n.3, p.429-451, 1994.

HOUGHTON, R. A. Aboveground forest biomass and the global carbon balance. Global

Change Biology, v.11, n.6, p. 945-958, 2005.

INTERGOVERNMENTAL PANEL ON CLIMATE CHANGE - IPCC. Land use, land use change, and forestry special report. Summary for Policymakers. Montreal: 2000.

LUYSSAERT, S. et al. Old-growth forests as global carbon sinks. Nature, v.455, p.213-215, 2008.

MELO, A. C. G.; DURIGAN, G. Fixação de carbono em reflorestamentos de matas ciliares o Vale do Paranapanema, SP, Brasil. Scientia Florestalis, n.71, p.149-154, 2006.

NASCIMENTO, H. E. M.; LAURANCE, W. F. Total aboveground biomass in central Amazonian rainforests: a landscape-scale study. Forest Ecology and Management, v.168, 1/3, p.311-321, 2002.

PAIXÃO, F. A. et al. Quantificação do estoque de carbono e avaliação econômica de diferentes alternativas de manejo em um plantio de eucalipto. Revista Árvore, v.30, n.3, p.411-420, 2006.
SANTOS, S. R. M.; MIRANDA, I. S.; TOURINHO, M. M. Estimativa de biomassa de sistemas agroflorestais das várzeas do rio juba, Cametá, Pará. Acta Amazônica, v. 34, n. 1, p.1-08, 2004.

SIERRA, C. A. et al. Total carbon stocks in a tropical forest landscape of the Porce region, Colombia. Forest Ecology and Management, v.243, n.2/3, p.299-309, 2007.

SOARES, C. P. B.; PAULA NETO, F.; SOUZA, A.L. Dendrometria e inventário florestal. $2^{\text {ed }}$. Viçosa, MG: Editora UFV, 2011. 272p.

TIEPOLO, G.; CALMON, M.; FERRETTI, A. R. Measuring and monitoring carbon stocks at the Guaraqueçaba Climate Action Project, Paraná, Brazil. In: Taiwan Forestry Research Institute. INTERNATIONAL SYMPOSIUM ON FOREST CARBON SEQUESTRATION AND MONITORING, 2002. p. 98-115. (Extension Serie, 153)

VOGEL, H. L. M.; SCHUMACHER, M. V.; TRÜBY, P. Quantificação da biomassa em uma Floresta Estacional Decidual Em Itaara, RS, Brasil. Ciência Florestal, v.16, n.4, p.419-425, 2006.

WATZLAWICK, L. F. et al. Fixação de carbono em Floresta Ombrófila Mista em diferentes estágios de regeneração. In: SANQUETA, C. R. et al. As florestas e o carbono. Curitiba: Universidade Federal do Paraná, 2002. Parte 8. p.153-173. 
